\title{
Arrhythmia Detection Algorithm using GoogLeNet and Generative Adversarial Network with Lifelog Signals
}

\author{
Siho Shin, Jaehyo Jung, Mingu Kang, Youn Tae Kim \\ IT Fusion Technology Research Center, Department of IT Fusion Technology, Chosun University, \\ 309 Pilmun-daero Dong-gu Gwangju 61452, Korea. \\ petruskim@chosun.ac.kr
}

Received: June 27, 2020. Revised: November 26, 2020. Accepted: December 16, 2020. Published: January 11, 2021.

\begin{abstract}
Arrhythmia is a cardiovascular disease with an irregular heartbeat, which can lead to a heart attack if it lasts for an excessive amount of time. Because the symptoms of arrhythmia occur irregularly, the heart needs to be monitored for a lengthy time period. This study suggests an arrhythmia diagnosis algorithm using GoogLeNet and a GAN. Because the algorithm proposed in this study can add to the number of data using a GAN, it can accurately diagnose an arrhythmic occurrence from measured lifelog over a short period of time. The classification of ECG data using GoogLeNet and a GAN showed an accuracy of approximately $\mathbf{9 9 \%}$.
\end{abstract}

Keywords-Arrhythmia, GoogLeNet, Generative Adversarial Network, Lifelog

\section{INTRODUCTION}

A heart rate indicates the rhythmic beating of the heart by electricity generated within the heart. A healthy individual has an active heart rate of 60 to 100 beats per minute [1]. When a person is exercising, nervous, or excited, the heart beats more quickly. In such cases, no problems generally occur. However, if the heart beats irregularly for no reason, it means there is a problem with the health of the heart. Such an occurrence is called arrhythmia.

If the heart rate is too fast, it is called tachycardia. If the heart rate is too slow, it is called bradycardia [2]. Tachycardia and bradycardia are serious symptoms that can cause cardiac arrest, and it is therefore extremely important to detect them in advance. Existing arrhythmia detection methods include early contraction detection of ventricles using the RR interval, detection using a discrete Fourier transform, and detection based on a Hilbert transformation [3-6]. In addition, although there are many other ways to detect arrhythmia, all methods require knowledge regarding the use of an electrocardiogram. Such measurements must also be carried out by experts.

To compensate for the aforementioned shortcomings, in this paper, an arrhythmia detection algorithm using GoogLeNet and a GAN is proposed. Because the algorithm proposed in this study adds ECG data using a GAN, there is no need to measure the ECG over a long period of time. In addition, optimal results can be obtained from a small ECG dataset.

\section{METHOD}

For arrhythmia detection, after designing a digital filter that minimizes the noise of the ECG signals, wavelet transforms are applied to extract the feature points for filtered ECG signals [7]. ECG data are then added using a GAN, and an arrhythmic occurrence is detected using GoogLeNet.

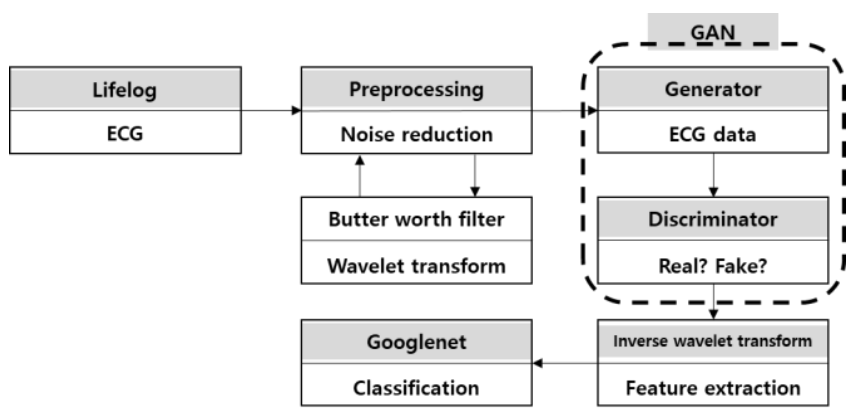

Fig. 1. Arrhythmia detection

\section{A. Preprocessing}

The noise contained in ECG signals varies in shape and size of the waveform owing to various environments. The causes of noise include contact with the electrodes, movement of the measurement cables, movements of the baseline by breathing, and noises caused by human movement. This noise results in poor accuracy in arrhythmia detection and must be minimized [8].
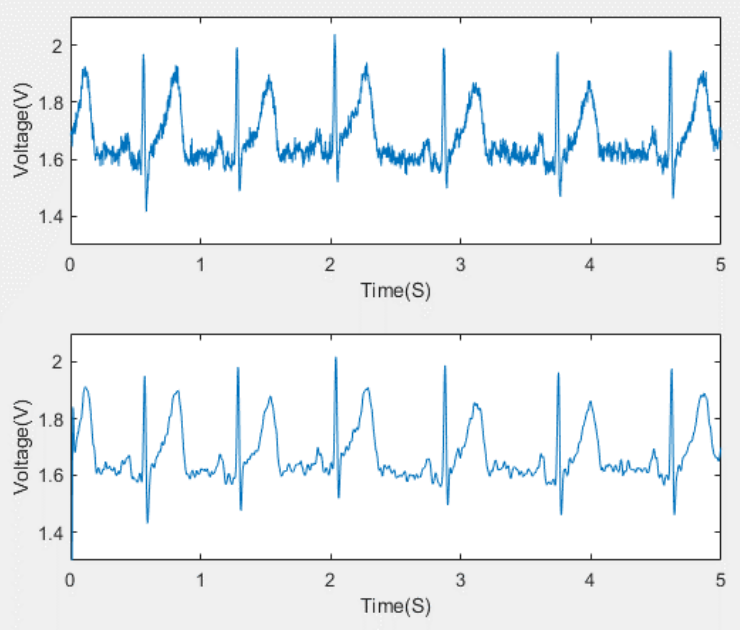

Fig. 2. Raw (top) and filter (bottom) signals

A baseline correction is a basic method of correcting the noise in an ECG signal.

ECG signals that oscillate irregularly from various noises are unsuitable for arrhythmia detection. An ECG signal can become suitable for arrhythmia detection by applying a frequency filter that changes the center axis of the ECG signal to a constant.

Subsequently, a Butterworth-type frequency filter is applied to remove any remaining noise from the ECG signal. 
The frequency bands of the filter are $0.5 \mathrm{~Hz}$ (HPF), $200 \mathrm{~Hz}$ (LPF), and $60 \mathrm{~Hz}$ (BSF) [9].

The wavelet transform is a signal processing method that converts a signal of the time domain into a signal of the frequency domain $[10,11]$. A wavelet transform is a useful tool for conducting an ECG signal analysis because it allows the extraction of feature points that cannot be extracted from the time domain.

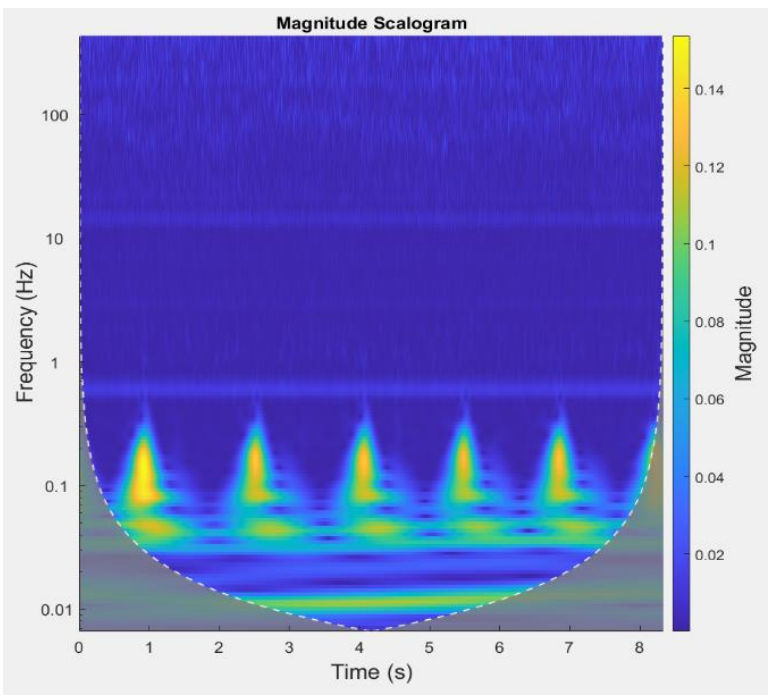

Fig. 3. Scalogram graph

\section{B. Data augment}

There are various electrocardiographic methods applied to an arrhythmia diagnosis, including 24-h electrocardiography and an exercise electrocardiogram, although all of them are cumbersome and take a long time to record [12-14]. Such measurements are not optimized for an arrhythmia diagnosis, which requires periodic checks of the heart condition. Therefore, a short-term arrhythmia diagnosis is required. To solve this problem, we need a method for randomly adding ECG data.

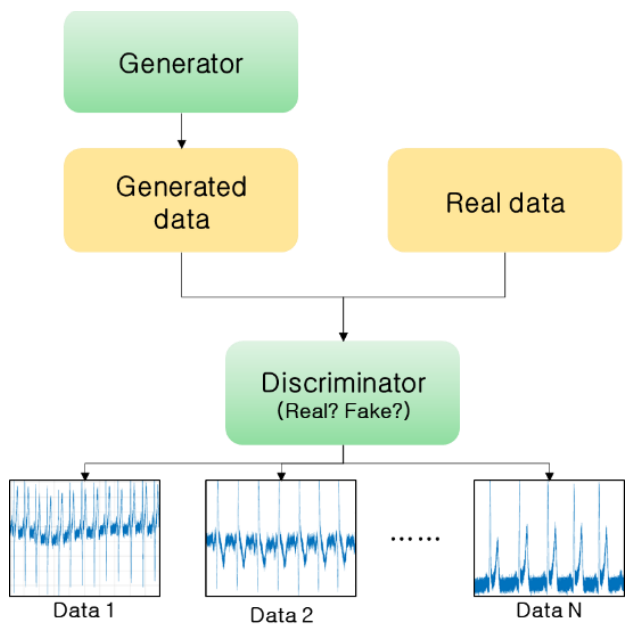

Fig. 4. GAN structure

A GAN is a neural network in which two neural network models compete to produce fake data such as if they were real data [15]. Both models are called generators and discriminators, and generate learn real data and generate fake data based on real data. The discriminator determines whether the data generated by the generators is real or fake. As the generators and discriminators compete against each other, fake data increasingly become real data. This process adds to the number of ECG data [16].

\section{Classification}

After removing noise from the ECG signal through a preprocessing, the threshold filter is applied to detect parameters such as the R wave, QRS complex, and R-R interval (RRI). Subsequently, a dataset is configured for arrhythmia detection.

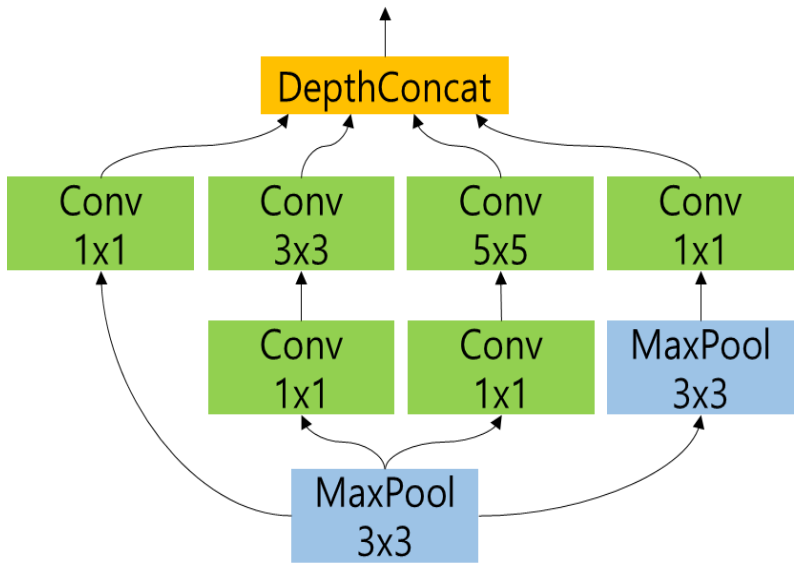

Fig. 5. Inception module of GoogLeNet

GoogLeNet is a neural network that improves its efficiency by deeply configuring the neural network and reducing the number of parameters. An arrhythmia is diagnosed by entering previously extracted angina-related parameters on GoogLeNet [17].

\section{RESULTS}

As a result of grouping ECG data using GoogLeNet and a GAN, $99 \%$ of the classification results were collected when the epoch value was over 20 . Once the algorithm exceeds 9 epochs, the accuracy converges to $100 \%$. This algorithm can be interpreted as having a high reliability even at a low number of epochs.

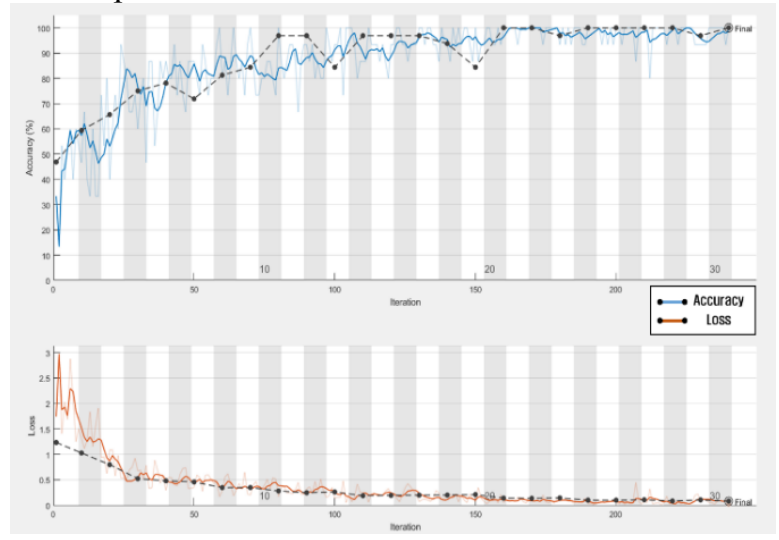

Fig. 6. Results of arrhythmia detection 
TABLE I. RESULTS OF ARRHYTHMIA DETECTION

\begin{tabular}{|c|c|c|c|c|c|c|}
\hline Epoch & Iteration & Time Elapsed & $\begin{array}{c}\text { Mini-batch } \\
\text { Accuracy }\end{array}$ & $\begin{array}{c}\text { Validation } \\
\text { Accuracy }\end{array}$ & $\begin{array}{c}\text { Mini- } \\
\text { batch } \\
\text { Loss }\end{array}$ & $\begin{array}{c}\text { Validation } \\
\text { Loss }\end{array}$ \\
\hline 1 & 1 & $0: 00: 09$ & $33.33 \%$ & $46.88 \%$ & 1.74 & 1.23 \\
\hline 2 & 10 & $0: 00: 40$ & $46.67 \%$ & $59.38 \%$ & 1.32 & 1.02 \\
\hline 3 & 20 & $0: 01: 12$ & $40.00 \%$ & $65.63 \%$ & 1.08 & 0.79 \\
\hline 4 & 30 & $0: 01: 43$ & $60.00 \%$ & $75.00 \%$ & 0.67 & 0.52 \\
\hline 5 & 40 & $0: 02: 14$ & $86.67 \%$ & $78.13 \%$ & 0.29 & 0.47 \\
\hline 7 & 50 & $0: 02: 45$ & $86.67 \%$ & $71.88 \%$ & 0.35 & 0.45 \\
\hline 8 & 60 & $0: 03: 17$ & $80.00 \%$ & $81.25 \%$ & 0.28 & 0.34 \\
\hline 9 & 70 & $0: 03: 49$ & $100.00 \%$ & $84.38 \%$ & 0.2 & 0.34 \\
\hline 10 & 80 & $0: 04: 21$ & $100.00 \%$ & $96.88 \%$ & 0.17 & 0.28 \\
\hline 12 & 90 & $0: 04: 52$ & $93.33 \%$ & $96.88 \%$ & 0.31 & 0.24 \\
\hline 13 & 100 & $0: 05: 24$ & $93.33 \%$ & $84.38 \%$ & 0.13 & 0.26 \\
\hline 14 & 110 & $0: 05: 55$ & $86.67 \%$ & $96.88 \%$ & 0.25 & 0.18 \\
\hline 15 & 120 & $0: 06: 26$ & $93.33 \%$ & $96.88 \%$ & 0.21 & 0.19 \\
\hline 17 & 130 & $0: 06: 58$ & $100.00 \%$ & $96.88 \%$ & 0.07 & 0.19 \\
\hline 18 & 140 & $0: 07: 29$ & $93.33 \%$ & $93.75 \%$ & 0.15 & 0.2 \\
\hline 19 & 150 & $0: 08: 02$ & $100.00 \%$ & $84.38 \%$ & 0.06 & 0.21 \\
\hline 20 & 160 & $0: 08: 35$ & $100.00 \%$ & $100.00 \%$ & 0.05 & 0.14 \\
\hline 22 & 170 & $0: 09: 06$ & $100.00 \%$ & $100.00 \%$ & 0.09 & 0.13 \\
\hline 23 & 180 & $0: 09: 37$ & $93.33 \%$ & $96.88 \%$ & 0.13 & 0.14 \\
\hline 24 & 190 & $0: 10: 12$ & $100.00 \%$ & $100.00 \%$ & 0.02 & 0.1 \\
\hline 25 & 200 & $0: 10: 45$ & $100.00 \%$ & $100.00 \%$ & 0.05 & 0.1 \\
\hline 27 & 210 & $0: 11: 46$ & $100.00 \%$ & $100.00 \%$ & 0.07 & 0.1 \\
\hline 28 & 220 & $0: 11: 46$ & $100.00 \%$ & $100.00 \%$ & 0.06 & 0.08 \\
\hline 29 & 230 & $0: 12: 17$ & $93.33 \%$ & $96.88 \%$ & 0.07 & 0.1 \\
\hline 30 & 240 & $0: 12: 47$ & $100.00 \%$ & $100.00 \%$ & 0.02 & 0.07 \\
\hline & & & & & & \\
\hline & & & & & \\
\hline & & & & & &
\end{tabular}

\section{CONCLUSION}

In this study, an algorithm for diagnosing an arrhythmia using GoogLeNet and GAN is proposed. Because ECG data measured over a short period of time make up a small number in the dataset, it is difficult to diagnose arrhythmia accurately. To compensate for this, ECG data were added using a GAN. The classification of ECG data using GoogLeNet and a GAN showed an accuracy of approximately $99 \%$. This algorithm shows a high accuracy even at a low number of epochs. These studies can be applied to the diagnosis of diseases requiring periodic monitoring. In addition, arrhythmia detection algorithms are expected to be used in various fields of healthcare and the Internet of Things.

\section{ACKNOWLEDGMENT}

This research was supported by the Mid-career Researcher Program (No. 2016R1A2B3009423) through NRF grant funded by the MSIT (Ministry of Science and ICT) and the Basic Science Research Program through the NRF funded by the Ministry of Education of Korea (No. 2018R1A6A1A 03015496).

\section{REFERENCES}

[1] Mayo Clinic, LIVESCIENCE, "What is a Normal Heart Rate?", 2018

[2] Victor Chang, Cardiac Research institue "Arrhythmia", 2020.

[3] VOLLMER, Marcus, "Arrhythmia classification in long-term data using relative RR intervals", In: 2017 Computing in Cardiology (CinC). IEEE, 2017, pp. 1-4.

[4] HUANG, Jingshan, et al, "ECG arrhythmia classification using STFT based spectrogram and convolutional neural network", IEEE Access, 2019, pp. 92871-92880.

[5] GUPTA, Varun; MITTAL, Monika, "R-peak based Arrhythmia Detection using Hilbert Transform and Principal Component Analysis", In: 2018 3rd International Innovative Applications of Computational Intelligence on Power, Energy and Controls with their Impact on Humanity (CIPECH), IEEE, 2018. pp. 1-4.
[6] Saudagar, Bhawna Jindal, "R Peak Detection with Diagnosis of Arrhythmia using Adaptive Filter and Hilbert Transform.", 2019.

[7] Markus H"oglinger, JOHANNES KEPLER UNIVERSITY "ECG Preprocessing", 2016.

[8] ROMERO, Francisco Perdigón, et al, "Baseline wander removal methods for ECG signals: A comparative study", arXiv preprint arXiv, 2018.

[9] Liu, Ming, et al. "Constructing a guided filter by exploiting the butterworth filter for ECG signal enhancement." Journal of Medical and Biological Engineering, 2018, pp. 980-992.

[10] XU, Xiaowen, et al, "Adaptive motion artifact reduction based on empirical wavelet transform and wavelet thresholding for the noncontact ECG monitoring systems", Sensors, vol. 19, 2019.

[11] SAHOO, Santanu, et al. Multiresolution wavelet transform based feature extraction and ECG classification to detect cardiac abnormalities. Measurement, vol. 108, pp. 55-66, May 2017.

[12] NELSON, Benjamin W.; ALLEN, Nicholas B. “Accuracy of consumer wearable heart rate measurement during an ecologically valid 24-hour period: intraindividual validation study", JMIR mHealth and uHealth, vol. 7, May 2019.

[13] VAIDYA, Gaurang Nandkishor, "Application of exercise ECG stress test in the current high cost modern-era healthcare system", Indian heart journal, 2017, pp. 551-555.

[14] PLESINGER, Filip, et al, "Automatic detection of atrial fibrillation and other arrhythmias in holter ECG recordings using rhythm features and neural networks", Computing in Cardiology (CinC) IEEE, 2017. p. 1 4.

[15] DELANEY, Anne Marie; BROPHY, Eoin; WARD, Tomas E. "Synthesis of Realistic ECG using Generative Adversarial Networks", arXiv preprint arXiv: 2019.

[16] FRID-ADAR, Maayan, et al, "Synthetic data augmentation using GAN for improved liver lesion classification", 2018 IEEE 15th international symposium on biomedical imaging (ISBI 2018), 2018, pp. 289-293.

[17] KIM, Jeong-Hwan, et al, "Assessment of electrocardiogram rhythms by GoogLeNet deep neural network architecture", Journal of healthcare engineering, 2019.

\section{Creative Commons Attribution License 4.0 (Attribution 4.0 International, CC BY 4.0)}

This article is published under the terms of the Creative Commons Attribution License 4.0 https://creativecommons.org/licenses/by/4.0/deed.en_US 